\title{
Veterinary Clinical Pathology
}

An International Journal of Laboratory Medicine

\section{Evaluation of the analytical variability of dipstick protein pads on canine urine}

\begin{tabular}{|r|l|}
\hline Journal: & Veterinary Clinical Pathology \\
\hline Manuscript ID & Draft \\
\hline Manuscript Type: & Original Article \\
\hline Date Submitted by the Author: & n/a \\
\hline Complete List of Authors: & $\begin{array}{l}\text { Giraldi, Marco; University of Milan, Department of Veterinary Medicine; } \\
\text { University of Milan, Veterinary Teaching Hospital } \\
\text { Paltrinieri, Saverio; University of Milan, Department of Veterinary Medicine; } \\
\text { University of Milan, Veterinary Teaching Hospital } \\
\text { Zatelli, Andrea; Medical Consultancy Services, }\end{array}$ \\
\hline Key Words: & Dogs, Proteinuria, Reagent strips, Urinalysis \\
\hline \multicolumn{2}{|c}{} \\
\hline
\end{tabular}


1 Evaluation of the analytical variability of dipstick protein pads on canine urine

2

3 Dipstick analysis of canine proteinuria

4

5 Marco Giraldi, ${ }^{a, b}$ DVM

6 Saverio Paltrinieri, ${ }^{a, b *}$ DVM PhD Dipl ECVCP

$7 \quad$ Andrea Zatelli ${ }^{\mathrm{c}}$, DVM PhD

8

$9{ }^{a}$ Department of Veterinary Medicine - University of Milan, Via Celoria, 10 - 20133

10 Milan, Italy

$11{ }^{b}$ Veterinary teaching hospital - University of Milan, Via dell'Università 6 - 26900 Lodi,

12 Italy

$13{ }^{c}$ Medical Consultancy Services, Ta’Xbiex, Malta

14

15

16 * Corresponding author: Dr. Paltrinieri

17 Department of Veterinary Medicine - University of Milan,

18 Via Celoria, 10 - 20133 Milan, Italy

19 Tel.: +390250318103.

20 E-mail address: saverio.paltrinieri@unimi.it

21 


\section{Abstract}

23 Background: The dipstick is a first line and inexpensive test to exclude the presence

24 of proteinuria in dogs. No information is available on the analytical variability of 25 dipstick analysis

26 Objectives: The aim of this study was to assess the analytical variability of two 27 dipsticks and the inter-operator variability in dipstick interpretation 28 Methods: Canine urine supernatants $(n=174)$ were analyzed with two commercially 29 available dipstick. Two observers evaluated each result blinded to the other observer 30 and to the results of the other dipstick. Intra and inter-assay variability were assessed on 5 samples (corresponding to the 5 different semi-quantitative results) tested for 10 consecutive times and 5 consecutive days, respectively. The variability between observer and between dipsticks was evaluated with Cohen's k test.

34 Results: Intra-assay repeatability was good ( $\leq 3 / 10$ errors), whereas inter-assay variability was higher (from $1 / 5$ to $4 / 5$ discordant results). The concordance between operators ( $k=0.68$ and 0.79 for the two dipsticks) and between dipsticks ( $k=0.66$ and

370.74 for the two operators) was good. However, one observer and one dipstick overestimated the results compared with the other observer or dipstick. In any case, discordant results accounted for a single unit of the semi-quantitative scale.

Conclusions: As for any other method, analytical variability may affect the semiquantification of urinary proteins with dipstick. Subjective interpretation of the pad and, to a lesser extent, intrinsic staining properties of the pads could affect the results. Further studies are warranted in order to evaluate the effect of this variability on clinical decision.

Keywords: Dogs, Proteinuria, Reagent strips, Urinalysis 


\section{Introduction}

48 In clinical practice proteinuria is defined as the increased amount of proteins in the 49 urine. The presence of persistent proteinuria of renal origin has a diagnostic and 50 prognostic value for chronic kidney disease (CKD) in dogs. The detection of 51 proteinuria is therefore a milestone in the management of dogs with CKD. ${ }^{1}$

52 The recommended methods to evaluate proteinuria in dogs is the quantitative evaluation of the urinary proteins and urinary creatinine, followed by the calculation of

54 the urinary protein to creatinine (UPC) ratio, that allows to correct the magnitude of 55 proteinuria by the dilution of urine. ${ }^{1,2}$ However, in clinical practice, dry reagent test strips (dipsticks) are rapid and inexpensive methods that allow, along with other urinary physico-chemical or cytological parameters, a first evaluation of the presence or absence of proteins in urine in a point-of-care setting. ${ }^{3}$ Proteins (mainly albumin), when present, react with the pad yielding a variable color change whose intensity is proportional to the protein concentration. Results are then expressed semiquantitatively, usually as negative, trace or $1+$ to $4+$ (corresponding, for most of the commercially available dipsticks to 15 to $2000 \mathrm{mg} / \mathrm{dL}$ of proteins), comparing the pad against the chart on the side of the dipstick package or, alternatively, loading the strip in an automated spectrophotometric reader.

Although the evaluation of protein excretion using the dipstick must be considered a screening test, erroneous interpretations of the pad may affect clinical decisions. In

67 human medicine information from quality assurance programs revealed that the rates

68 of misclassification of one and two scores above or below the expected value were

$699.7 \%$ and $2.3 \%$, respectively, ${ }^{4}$ and that the intra- and inter-observer agreement is 70 moderate $(k=0.53)$ to very good $(k=1) .{ }^{5}$ Moreover, the use of automated reader is 71 recommended to minimize the observer-related errors ${ }^{6}$ despite automated readings 
72 only slightly improved the reproducibility of dipstick analysis. ${ }^{5}$ In veterinary medicine,

73 although similar results are likely, and quality control programs for urinalysis and

74 dipstick tests are recommended, ${ }^{2}$ little is known about the influence of inter-operator

75 variability on the analytical variability of dipstick testing or about the intrinsic

76 performances of the dipstick from different manufacturers, that may have their

77 peculiar analytical sensitivity and range or different semi-quantitative interpretation

78 charts.

79 Therefore, the aim of this study was to assess the analytical variability of dipstick

80 analysis in the evaluation of the presence/absence of proteins in canine urine,

81 through the comparison of results obtained using two commercially available

82 dipsticks by two different independent operators.

83

84 Materials and Methods

85 A total of 174 canine urine supernatants were included in this study.

86 Urine samples were collected over a period of 12 months from dogs of different age,

87 sex and breed, presented for diagnostic investigation at the internal medicine unit of

88 the Department of Veterinary Medicine (DIMEVET, University of Milan), by mean of

89 cystocenthesis $(n=113)$, free catch $(n=47)$ or unspecified method $(n=14)$. Samples

90 were collected for diagnostic purposes under informed consent of the owner and

91 therefore, according to the institutional Ethical Committee (deliberation number:

92 2/2016) a formal approval of the study from the Institutional Animal Care and Use

93 Committee was not necessary.

94 Since this was a validation study not focused on the impact of the results on the

95 clinical diagnosis, samples were included irrespective of health status of the dogs. 
All urine samples underwent complete urinalysis (including USG, dipstick evaluation and sediment examination) within two hours from collection. In order to perform sediment analysis, $5 \mathrm{~mL}$ of sample were placed in sterile conical $10 \mathrm{~mL}$ tube and centrifuged 5 minutes at $1250 \mathrm{rpm}(450 \mathrm{G})$. Supernatants were aliquoted in a $1.5 \mathrm{~mL}$ tube and stored at $-20^{\circ} \mathrm{C}$ within 4 hours from collection for a maximum of 12 months. At the time of analysis, supernatants were gently thawed by transferring tubes at $4^{\circ} \mathrm{C}$ the day before analysis and then at room temperature one hour before analysis. Each sample was tested with two commercially available dipsticks (Dipstick 1: U-11 Urine Strips, Mindray, Shenzhen, China; Dipstick 2: Multistix 10 SG Reagent Strips, Siemens, Siemens Healthcare Diagnostics Inc, Tarrytown, NY /Siemens Healthcare Diagnostics, Eschborn, Germany) after a preliminary assessment of intra- and interassay variability of each dipstick (see below). Moreover, each dipstick was evaluated by two operators with similar experience in urinalysis procedures. In each analysis, the dipstick was kept out from the case no more than 2 minutes before the use. Fifty microliters of urines supernatant were applied with a dispensable pipette only on the protein pad and, in order to prevent crosscontamination by dyes from close pads the contact of urine with adjacent pads was avoided; then, excess urine was gently discarded hitting the dipstick on a clean paper towel.

In order to avoid bias in interpretation of the second dipstick by each operator and to avoid excess time between urine application and reading, samples were analyzed in batches ( 8 samples per batch), thus allowing the evaluation within $60-120$ seconds, as recommended by the manufacturer's instruction of both the dipstick kits, and urine samples were applied to the second dipstick in a different random order compared with that used for the first dipstick. Each operator interpreted the dipsticks results 
121 blinded to the results of the other operator. Also, due to the different random order 122 described above, the interpretation of the second dipstick was blinded to results of 123 the first dipstick.

124 Interpretation of each dipstick was performed by comparing the color of the protein 125 pads with the corresponding color chart provided by each manufacturer.

126 Because, as expected, some samples yielded a color reaction with a chromatic 127 intensity not identical to those proposed on the chart, the following reading method 128 was chosen: each pad was compared with one reference color block at a time; when 129 an almost perfect match between the pad and the block on the chart was identified, 130 the corresponding result was assigned (i.e negative or trace - N/T - or positive: $1+$, $1312+, 3+$ or $4+$ ); when the color intensity was intermediate between two blocks on the 132 chart, the results corresponding to the nearest reference color blocks (lighter or 133 darker) was assigned. However, in these cases, the samples were also recorded as 134 "difficult".

\section{Intra-assay and inter-assay precision}

137 Five urine samples yielding results corresponding to the 5 different semi-quantitative 138 scores of proteinuria (namely $\mathrm{N} / \mathrm{T}=<30 \mathrm{mg} / \mathrm{dL}, 1+=30 \mathrm{mg} / \mathrm{dL}, 2+=100 \mathrm{mg} / \mathrm{dL}, 3+$ $139=300 \mathrm{mg} / \mathrm{dL}, 4+=\geq 2 \mathrm{~g} / \mathrm{dL}$ ) were selected and used for analytical precision tests.

140 For intra-assay evaluation, the 5 samples were tested with both dipsticks 10 141 consecutive times.

142 Then, inter-assay variability was assessed testing the same samples 5 times in 5 143 consecutive days by both dipsticks, storing samples at $4^{\circ} \mathrm{C}$ overnight between the 144 evaluations. 
145 For both intra- and inter-assay evaluations, interpretation of pads was performed with

146 the same method described for the whole set of samples.

147 For each semi-quantitative score, numbers of different results were counted and the

148 percentage of the results lower (underestimation) or higher (overestimation) than the

149 first reading were calculated.

150 Imprecision was expressed as the percentage of different results out of the sum of

151 the two operators (i.e 20 reading/results for intra-assay evaluation and 10 results for

152 inter-assay evaluation)

153

\section{Statistical analysis}

155 Concordance between operators and between dipsticks was tested with Cohen's 156 Kappa test $^{7}$ and was calculated either for the whole set of results $(\mathrm{N} / \mathrm{T}, 1+, 2+, 3+$, $1574+$ ) or grouping results as $\leq 1$ and $\geq 2$.

158 Moreover, for the evaluation of the concordance between dipsticks, the results of the 159 two operators were combined in order to reach a consensus and, in case of 160 discordant results, further intermediate categories were created $(\mathrm{N} / \mathrm{T}-1+, 1+-2+$, $1612+-3+, 3+-4+)$.

162 The k coefficients were used to define the concordance as follows: $0.00-0.20$, $1630.21-0.40,0.41-0.60,0.61-0.80$ and $0.81-1.00$ represented poor, fair, moderate, 164 good and very good concordance ${ }^{8}$, respectively.

165 In order to quantify the rate of discordant results at different level of positivity, the 166 number of discordant sample yielded between two scores (e.g. between N/T and 1+ 167 or between $1+$ and $2+$ etc.) were counted and the percentage was calculated out of 168 the total number of samples found among the two scores evaluated. 
169 Descriptive statistics were performed with Excel software and the Analyze-it

170 statistical software (Analyse-it Software Ltd, Leeds, West Yorkshire, England) was

171 used to assess the level of concordance (Cohen's k)

172

173 Results

174 Intra-assay variability

175 Dipstick 1 always provided the same results recorded at first reading, except in two

176 cases: operator 1 overestimated one $3+$ sample (10\%) and Operator 2

177 underestimated one 2+ sample (10\%) Difficult interpretations were more frequent for

178 Operator $1(5 / 10$ at $1+; 2 / 10$ at $2+$ and $3+)$ than for Operator $2(4 / 10$ at $2+)$.

179 Using Dipstick 2, Operator 1 overestimated one $1+$, one $2+$ and one $3+$ sample (10\%

180 each), while Operator 2 underestimated three $2+$ and three $3+$ samples (30\% each).

181 Only Operator 1 recorded difficult interpretations (3/10 at $1+$ and $2+$ and $1 / 10$ at $3+)$.

182

183 Inter-assay variability

184 With Dipstick 1, Operator 1 overestimated one 1+ sample (20\%) and Operator 2

185

overestimated one $1+$ sample $(20 \%)$ and underestimated three $2+$ samples $(60 \%)$

186 and one 3+ sample (20\%). Difficult interpretations were rare either for Operator 1

$187(1 / 10$ at N/T and at $2+)$ or for Operator $2(1 / 10$ at $3+)$.

188 Imprecision was more frequent for Dipstick 2: Operator 1 overestimated four 1+

$189(40 \%)$ and four $3+$ samples (80\%), while Operator 2 overestimated one $1+(10 \%)$,

190 one $2+(10 \%)$ and three $3+$ samples (30\%). Only Operator 1 recorded difficult

191 interpretations $(1 / 10$ at $2+$ and $3+)$.

192

193 Analysis of samples 
194 Samples covered all possible results of the dipsticks but results with scores lower 195 than $2+$ were more frequent for both the dipsticks, as follows.

196 Using Dipstick 1, samples recorded as N/T, 1+, 2+, 3+ and 4+, were respectively 96, $19750,17,10$ and 1 for Operator 1 and 112, 35, 16, 10 and 1 for Operator 2 (Table 1).

198 Operators 1 and 2 recorded $23(13,2 \%)$ and $22(12.6 \%)$ difficult interpretations, 199 respectively. These uncertain interpretations were mostly between negative and 200 trace or between trace and $1+$ either for Operator $1(n=10$, and $n=10$, respectively) or 201 Operator 2 ( $n=12$ and $n=6$, respectively). The total number of samples that were 202 difficult for both operators was 39 (22.4\%).

203 Using Dipstick 2, samples recorded as N/T, 1+, 2+, 3+ and 4+, were respectively 84, $20453,19,12$ and 6 for Operator 1 and 105, 37, 17, 11 and 4 for Operator 2 (Table 1). 205 Operators 1 and 2 recorded $23(13,2 \%)$ and $32(18.4 \%)$ difficult interpretations, 206 respectively. These uncertain interpretations were mostly between negative or trace, 207 between trace and $1+$ and between $1+$ and $2+$ either for Operator $1(n=7, n=8$, and $208 \mathrm{n}=6$, respectively) or $2(\mathrm{n}=11, \mathrm{n}=14$ and $\mathrm{n}=3$, respectively). The total number of 209 samples that were difficult for both operators was $46(26.4 \%)$.

210

\section{Concordance between operators}

212 The concordance between operators was "good" for both dipsticks, and slightly 213 higher for Dipstick $1(k=0.79)$ than for Dipstick $2(k=0.68)$. Discordant results were 214 found mostly at lower protein concentrations for dipstick 1 (N/T vs $1+: 11 \%$ of 215 misclassifications, $1+$ vs $2+: 6 \%$ of misclassifications; $2+$ vs $3+: 8 \%$ of 216 misclassifications) but at all the protein concentrations for dipstick 2 (N/T vs 1+: $17 \%$ 217 of misclassifications, $1+$ vs $2+: 14 \%$ of misclassifications; $2+$ vs $3+: 12 \%$ of 
218 misclassifications; $13 \%$ of misclassifications). Moreover, for both the dipsticks

219 Operator 1 tended to provide higher scores compared with Operator 2.

220 When results were grouped as $\leq 1+$ and $\geq 2$, concordance improved consistently to

221 the "very good" category (Dipstick 1 k=0.94; Dipstick $2 \mathrm{k}=0.87$ ) and only 3 and 7

222 discordant results were misclassified with Dipstick 1 and Dipstick 2, respectively.

223

\section{Concordance between dipsticks}

225 The concordance between the two dipsticks was "good" for both operators (Operator

$2261 \mathrm{k}=0.66$; Operator $2 \mathrm{k}=0.74)$. Similarly to the inter-operator variability described

227 above, discordant results were more frequent for Observer 1 (N/T vs $1+: 12 \%$ of

228 misclassifications, $1+$ vs $2+: 16 \%$ of misclassifications; $2+$ vs $3+: 30 \%$ of

229 misclassifications; $3+$ vs $4+: 45 \%$ of misclassifications) than for Observer 2 (N/T vs

$2301+: 11 \%$ of misclassifications, $1+$ vs $2+: 11 \%$ of misclassifications; $2+$ vs $3+: 17 \%$ of

231 misclassifications; $3+$ vs $4+: 27 \%$ of misclassifications)

232 When results were grouped as $\leq 1+$ and $\geq 2+$, concordance improved to "very good"

233 category ( $\mathrm{k}=0.83$ and $=0.90$ for Operator 1 and Operator 2, respectively), showing 9

234 and 5 samples misclassified by dipsticks ( $\geq 2$ using Dipstick 2 and $\leq 1$ using Dipstick

235 1) with Operator 1 and Operator 2, respectively.

236 Overall Dipstick 2 tended to provide higher scores compared with Dipstick 1.

237 Concordance between dipsticks using the consensual agreement between operators

238 (Table 2) was defined as "moderate" (k=0.59). Misclassifications were $22.5 \%$ in the

239 interval $\mathrm{N} / \mathrm{T}$ vs $1+, 42.8 \%$ in $1+$ vs $2+, 66.6 \%$ in $2+$ vs $3+$ and $66.6 \%$ in $3+$ vs $4+$.

240 Again, grouping results as $<1,1-2,>2$, concordance improved to "good" ( $k=0.76)$.

242 Discussion 
243 This study, using two commercially available dipsticks and two operators,

244 demonstrated that a variable imprecision in the evaluation of the concentration of 245 urinary proteins exist. The study design adopted to assess the analytical variability 246 (e.g. application of urine by the same operator, disposal of urine only on the protein 247 pad, analysis of 8 samples per batch, randomization of readings etc) prevented the 248 effect of other possible sources of error and variability such as oxidation of dipsticks, 249 insufficient amount of urine on the pad, excess urine with contamination from other 250 pads, colour changes due to delay of readings, interpretative biases due to the 251 sequential analysis of samples by the same operator, etc. ${ }^{4,9,10}$

252 Intra-assay imprecision, however, was similar in magnitude to that reported in human 253 medicine where the reproducibility of visual reading was $68-85 \%,{ }^{11}$ and was variable 254 between the operators. As for any other test, ${ }^{12}$ inter-assay variability was even 255 higher. Nevertheless, this linter-assay variability has a low clinical importance 256 because rarely repeated readings are performed during different days.

257 The imprecision may depend on intrinsic characteristics of the pads, on the visual 258 perception of the operators, on environmental factors (e.g. different light during days) 259 or, as regards inter-assay variability, on changes of $\mathrm{pH}$ or of protein concentration 260 induced by refrigeration, as shown in studies on the protein to creatinine ratio. ${ }^{13}$ 261 However, the dipstick is analytically less sensitive than wet chemistry and no effects 262 of storage on $\mathrm{pH}$ were reported in $\operatorname{dogs}^{14}$ and therefore storage artifacts are unlikely. 263 Moreover, imprecision tests evidenced a high frequency of samples that were difficult 264 to interpret, especially at low scores (i.e. N/T and 1+) and with Dipstick 2. For both 265 the dipsticks, the two operators found difficulty with different samples. This points out 266 that the difficult interpretation was operator-dependent instead of sample- or dipstick267 dependent. However, to our impression, the two dipstick had slight differences in the 
268 colour of the pad before the application of urines and in the hue after reaction with

269 the same samples (especially at low protein concentration). This difference may

270 complicate the interpretation of results. Whether sample-dependent factors (e.g.

271 physical or chemical properties of urine such as urine dilution or presence of

272 pigments) could affect the agreement between dipsticks needs further evaluation.

273 This study showed also that the inter-operator concordance was not perfect, due to

274 discordant results at all the levels of positivity. The degrees of concordance recorded

275 in this study were similar to that found in people, where a k coefficient of 0.82 was

276 found ${ }^{5}$ but lower than that reported in dogs $(k=0.92){ }^{15}$

277 The majority of the discordant results were due to an overestimation of results by one 278 operator compared with the other. Also in human medicine a tendency to 279 overestimate or underestimate protein pads by single operators was demonstrated, ${ }^{11}$ 280 likely due to the different visual perception mentioned above. Moreover, although the 281 reading method was standardized between the operators, each operator could 282 consistently perceive as closer to the lower or the higher score the color reactions 283 that were intermediate to those shown on the chart, as already demonstrated. ${ }^{10}$

284 Similarly to the results of inter-operator variability, sub-optimal concordances 285 between dipsticks were found with both operators. Interestingly, Dipstick 2 tented to 286 provide higher scores compared with Dipstick 1, and about a quarter of samples $\geq 2+$ 287 by Dipstick 2 were classified as $1+$ by Dipstick 1 . This result points out that, although 288 the two manufactures declared the same analytical sensitivity $(15 \mathrm{mg} / \mathrm{dL})$ and 289 reported equal protein concentrations for the 4 blocks on the chart, slight differences 290 in biochemical reaction may exist between different commercially available dipsticks. 291 According to a previous study in dogs, ${ }^{16}$ samples with negative dipstick are likely non 292 proteinuric, samples with 2+ or more are likely proteinuric and samples with 1+ may 
293 or may not be proteinuric depending on the USG. Therefore, misclassification of

294 samples as $\mathrm{N}$ or $1+$ and $1+$ or $2+$ could be of clinical significance, while

295 misclassification of samples with $2+, 3+$, or $4+$ may be less relevant on a clinical

296 point of view, since the calculation of the UPC ratio, that is more accurate that

297 dipstick, is recommended for any sample with results $\geq 2+$.

298 The increase of agreement grouping results as $\leq 1+$ or $\geq 2+$ showed that the inter-

299 operator variability decreases. In other words, samples with more than $2+$, likely

300 corresponding to proteinuric dogs according to a previous study, ${ }^{16}$ can be correctly

301 identified independently on the operator, while this results confirms that

302 discrepancies mainly occurred between $\mathrm{N} / \mathrm{T}$ results and $1+$, that according to the

303 study cited above should be considered respectively as definitely on proteinuric and

304 dubious, i.e. proteinuric or not depending on the USG. In practical terms this may

305 indicate that the analytical and inter-operator variability does not affect the sensitivity

306 of the dipstick but it may affect the specificity of the method. Therefore, further

307 research on samples with known USG and UPC ratio, which were not available in all

308 the cases included in the present study, is needed in order to evaluate the diagnostic

309 performances of the two dipsticks as well as to understand which of the two dipsticks

310 employed in this study is more accurate and which over- or underestimate proteinuria

311 compared with the UPC ratio.

312 Interestingly, in any case errors were higher than one score. Studies in human

313 medicine reported that errors higher than one score are possible in clinical practice

314 and may account up to $2.4 \%{ }^{4,9,11}$ However, those studies evaluated not only the

315 analytical variability (as in our study) but also the effect of preanalytical and

316 postanalitycal errors on variability. Evaluation of such a variability was beyond the

317 aims of this study but it could be speculated that also in veterinary practice, 
318 preanalytical errors may occur and, along with the analytical variability reported in the

319 present study, may induce misclassifications higher than one score of positivity.

320 In conclusion, although dipstick is considered simple and intuitive test, analytical

321 variability may affect the interpretation of results, as well as for any other diagnostic

322 tests. Both the imprecision and the difficulty of interpretation may depend either on

323 intrinsic factors of the pads or on different capability of the operators. The effect of

324 these variables could be considerable in misclassification of samples between two

325 contiguous scores at any level of positivity but misclassification of results between

$326 \mathrm{~N} / \mathrm{T}, 1+$ and $2+$ could be of clinical significance and therefore should be interpreted

327 with caution and confirmed with more sensitive methods such as the UPC ratio.

328 Further studies are warranted in order to evaluate whether automated readers may

329 reduce this variability, or to determine the accuracy of these dipsticks in comparison

330 with a gold standard method such as the UPC ratio and the effect of the variability

331 quantified in this study on clinical decisions.

332

333 Conflict of interest statement

334 None of the authors of this paper has a financial or personal relationship with other 335 people or organizations that could inappropriately influence or bias the content of the 336 paper.

338 Acknowledgements:

339 The Authors are grateful to Prof. Paola Scarpa that provided most of the samples

340 included in this study and Dr. Tiziana Vitiello that performed routine urinalysis.

342 References 
343 1. Grauer GF. IRIS: Proteinuria. Available at: http://www.iris-

344 kidney.com/education/proteinuria.html. Accessed January 22th, 2016.

345 2. Gunn-Christie RG, Flatland B, Friedrichs KR, et al. ASVCP quality assurance 346 guidelines: control of preanalytical, analytical, and postanalytical factors for 347 urinalysis, cytology, and clinical chemistry in veterinary laboratories. Vet Clin $348 \quad$ Pathol. 2012;41:18-26

349 3. Stockham SL, Scott MA. Fundamentals of veterinary clinical pathology. 2nd ed. $350 \quad$ Ames, IA: Wiley-Blackwell; 2008:415-494

351 4. Tighe P. Laboratory-based quality assurance program for near-patient urine 352 dipstick testing, 1990-1997: development, management and results. Br J Biomed 353 Sci. 1999;56:6-15.

354 5. Winkens RA, Leffers P, Degenaar CP, Houben AW. The reproducibility of 355 urinalysis using multiple reagent test strips. Eur J Clin Chem Clin Biochem. $356 \quad 1991 ; 29: 813-818$.

357 6. European Confederation of Laboratory Medicine. European urinalysis guidelines. $358 \quad$ Scand J Clin Lab Invest Suppl. 2000;231:1-86.

359 7. Kouri T, Laippala P, Kutter D, Gant V. Quality specifications for ordinal scale 360 measurements with multiproperty (multiple) urine test strips. Scand J Clin Lab $361 \quad$ Invest. 1999;59:523-526

362 8. Landis JR, Koch GG. The Measurement of Observer Agreement for Categorical 363 Data. Biometrics. 1977;33:159-174.

364 9. Tighe P. Urine dry reagent strip "error" rates using different reading methods. 365 Accred Qual Assur. 2000;5:488-490.

366 10. Berg B, Hellsing K, Jagenburg R, Kallner A. Guidelines for evaluation of reagent 367 strips. Exemplified by analysis of urine albumin and glucose concentration using 
visually read reagent strips. International Federation of Clinical Chemistry, Scientific Division. Scand J Clin Lab Invest. 1989;49:689-699.

11. Rumley A. Urine dipstick testing: comparison of results obtained by visual reading and with the Bayer CLINITEK 50. Ann Clin Biochem. 2000;37:220-221.

12. Westgard JO. Basic method validation. 2nd ed. Madison, Wis: Westgard QC Inc, 2003.

13. Rossi G, Giori L, Campagnola S, Zatelli A, Zini E, Paltrinieri S. Evaluation of factors that affect analytic variability of urine protein-to-creatinine ratio determination in dogs. Am J Vet Res. 2012;73:779-788

14. Froom P, Bieganiec B, Ehrenrich Z, Barak M. Stability of common analytes in urine refrigerated for $24 \mathrm{~h}$ before automated analysis by test strips. Clin Chem. 2000;46:1384-6.

15. Bauer N, Rettig S, Moritz A. Evaluation the Clinitek status ${ }^{\mathrm{TM}}$ automated dipstick analysis device for semiquantitative testing of canine urine. Res Vet Sci. $2008 ; 85: 467-472$

16. Zatelli A, Paltrinieri S, Nizi F, Roura X, Zini E. Evaluation of a urine dipstick test for confirmation or exclusion of proteinuria in dogs. Am J Vet Res. 2010;71:235240. 


\section{Tables and Figures}

389 Table 1 Contingency table of the 174 canine urine samples assayed with dipstick 1

390 (above) and dipstick 2 (below) and interpreted by the two operators. Concordant

391 results are in bold, discordant results are in italics

392

\section{Operator 2}

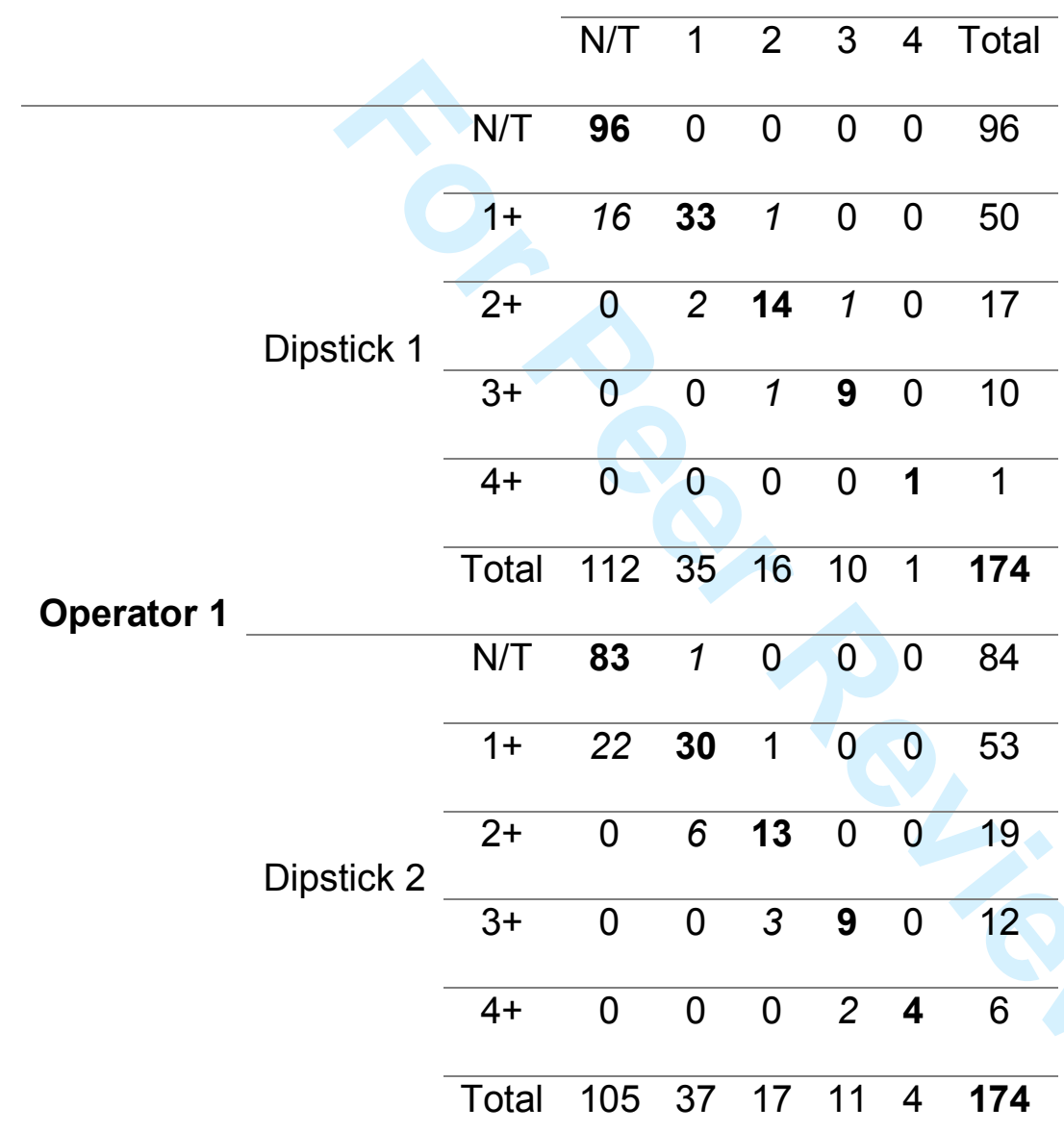


Table 2 Raw data of agreement between the two dipsticks in scoring protein pads using the results of the consensus between

\section{Dipstick2}

\begin{tabular}{|c|c|c|c|c|c|c|c|c|c|c|c|}
\hline & & & & & & & & & & & \\
\hline & & $\mathrm{N} / \mathrm{T}$ & $\mathrm{N} / \mathrm{T}$ or $1+$ & $1+$ & $1+$ or $2+$ & $2+$ & $2+$ or $3+$ & $3+$ & $3+$ or $4+$ & $4+$ & Total \\
\hline \multirow{9}{*}{ 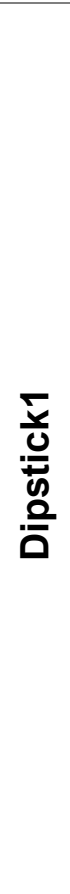 } & $\mathrm{N} / \mathrm{T}$ & 82 & 11 & 3 & 0 & 0 & 0 & 0 & 0 & 0 & 96 \\
\hline & $\mathrm{N} / \mathrm{T}$ or $1+$ & 0 & 9 & 7 & 0 & 0 & 0 & 0 & 0 & 0 & 16 \\
\hline & $1+$ & 1 & 3 & 20 & 7 & 2 & 0 & 0 & 0 & 0 & 33 \\
\hline & $1+$ or $2+$ & 0 & 0 & 0 & 0 & 3 & 0 & 0 & 0 & 0 & 3 \\
\hline & $2+$ & 0 & 0 & 0 & 0 & 8 & 3 & 3 & 0 & 0 & 14 \\
\hline & $2+$ or $3+$ & 0 & 0 & 0 & 0 & 0 & 0 & 2 & 0 & 0 & 2 \\
\hline & $3+$ & 0 & 0 & 0 & 0 & 0 & 0 & 4 & 1 & 3 & 8 \\
\hline & $3+$ or $4+$ & 0 & 0 & 0 & 0 & 0 & 0 & 0 & 1 & 0 & 1 \\
\hline & $4+$ & 0 & 0 & 0 & 0 & 0 & 0 & 0 & 0 & 1 & 1 \\
\hline
\end{tabular}




$\begin{array}{lllllllllll}\text { TOTAL } & 83 & 23 & 30 & 7 & 13 & 3 & 9 & 2 & 4 & 174\end{array}$

